graphiques spéciaux. Ces procédures sont judicieuses pour la protection du patient dans le domaine de la médecine de la transplantation. Mais dans la pratique du médecin de famille?

A l'avenir, devrai-je réanimer le vieux grandpère sur son banc de manière préventive et envoyer le chasseur en état d'hypothermie en ambulance au centre hospitalier jusqu'à ce qu'on ait établi si des organes peuvent être prélevés sur leurs corps? Dois-je «maintenir en vie» la jeune femme atteinte d'un cancer et détentrice inattendue d'une attestation de donneur, en la réanimant jusqu'au prélèvement éventuel d'organes?

Mes considérations peuvent sembler tirées par les cheveux, mais elles ne le sont pas aussi longtemps que le domaine d'application de la loi sur la transplantation n'est pas réglé dans une loi en fonction de la personne. L'article 2 dit seulement: «La présente loi s'applique à toute utilisation d'organes, de tissus ou de cellules d'origine humaine ou animale ainsi que de produits issus de ceux-ci (transplants standardisés) destinés à être transplantés sur l'être humain.» Si l'on limite le domaine d'application, ce qui serait judicieux du point de vue du médecin de famille, nous aurons effectivement deux réglementations juridiques concernant le décès, à savoir la «mort normale» et la «mort liée à une transplantation». Si la loi sur la transplantation devait, à l'avenir, être applicable de manière générale - ainsi que la révision de la loi cantonale bernoise le prévoit -, alors le médecin de famille ne serait plus d'aucune utilité dans le diagnostic du décès. Le voulons-nous?

\section{Littérature}

1 Canton de Berne. Loi sur la santé publique (modification) 24.10.2006.

2 Loi fédérale sur la transplantation d'organes, de tissus et de cellules (loi sur la transplantation) du 8.10.2004.

3 SAMW. Diagnostic de la mort dans le contexte de la transplantation d'organes. BMS 2005;86: 1871-1883.
Hanspeter Kuhn, avocat, secrétaire général adjoint de la FMH
Correspondance:

Service juridique de la FMH

Case postale 170

CH-3015 Berne

Tél.: 0313591111

Fax:031359 1112

lex@fmh.ch

\title{
La constatation des décès dans le cas «normal» incombe encore et toujours aux cantons
}

\section{Le Conseil fédéral réglera uniquement la question de la constatation des décès pour les transplantations}

La loi sur la transplantation entrera en vigueur dans le courant de l'année 2007. Dans ce contexte, le Conseil fédéral fixera par voie d'ordonnance les procédures complexes visant à constater la mort cérébrale. En revanche, la constatation des décès hors des cas de transplantation d'organe se fera toujours selon les règles en vigueur depuis des décennies et que l'on enseigne également aux étudiants en médecine dans le cadre de la médecine légale. Pour ces cas-là, ce sont les cantons qui restent compétents sur le plan formel pour la réglementation de la constatation des décès.

\section{Historique de la loi sur la transplantation} Le Conseil fédéral a demandé un avis de droit sur les questions relatives à la mort au Professeur Guillod de l'Institut de droit de la santé de l'Université de Neuchâtel, avis que le CF a ensuite résumé comme suit dans son message concernant la loi sur la transplantation:

Définition de la mort par le législateur

La définition de la mort a des effets pour chacun et non pas seulement pour certaines catégories de personnes ou pour les médecins. Cette définition doit être valable dans tous les cas: il n'y a qu'une seule mort et juridiquement, elle doit être la même pour tous (à cet égard, les procédures en vue de sa détermination peuvent être différentes selon le contexte). C'est pourquoi la tâche de définir la mort incombe $\mathrm{au}$ législateur et non pas au juge ou à une réunion d'experts (p. ex. l'ASSM ou la commission nationale d'éthique).

[...] De préférence, la définition de la mort devrait être insérée dans le CC et non pas dans la loi sur la transplantation. Cela soulignerait qu'il n'y a qu'une seule mort déployant des effets erga omnes.

Selon la jurisprudence du Tribunal fédéral, le concept de «mort cérébrale» est compatible avec la Constitution fédérale, singulièrement avec le droit fondamental à la vie.

Outre la définition de la mort, il faut examiner les aspects suivants en vue d'une réglementation:

- La détermination du moment du décès;

- Le statut des médecins appelés à constater le décès;

- La procédure de constatation du décès: il s'agit de règles scientifiques dont l'élaboration peut être déléguée à des organismes privés ou publics, p. ex. l'OFSP ou l'ASSM. (Extrait du message concernant la loi sur la transplantation, FF 2001, p. 88)

De l'avis de droit du Prof. Guillod, le Conseil fédéral a tiré les principes fondamentaux suivants:

$$
\begin{aligned}
& \text { «1.3.3.7.3 Principes à la base de la réglementation } \\
& \text { légale } \\
& {[\ldots]} \\
& \text { - La définition de la mort et le critère de décès } \\
& \text { doivent s'appliquer à toutes les personnes: il }
\end{aligned}
$$


1 Cf. par ex. U. Zollinger, K. Hartmann; Devoirs et obligations des médecins en matière d'annonce aux autorités judiciaires et policières; Réglementations légales non uniforme en Suisse en cas de décès, lésions corporelles et délits sexuels (en allemand seulement); Bulletin des médecins suisses. 2001;82(26): 1384-92 [trad. FMH]: «Tout décès ou découverte de corps doit, en vertu du Code civil, être annoncé à l'officier d'état civil compétent, afin que ce dernier puisse l'inscrire au registre des décès. Pour ce faire, il est nécessaire, conformément à l'article 82 de l'Ordonnance sur l'état civil de disposer d'un certificat de décès établi, rempli et signé par le médecin après qu'il a procédé personnellement à l'examen de la personne décédée (examen du cadavre). Par la signature de ce document important pour l'autorité d'état civil et pour les héritiers, le médecin confirme avec certitude la mort et l'identité de la personne dont il a pu déterminer ou supposer le moment du décès. [...]

La répartition entre mort naturelle d'une part, et mort extraordinaire, c'est-à-dire décès non naturel ou peu clair, d'autre part, permet, si elle est effectuée de manière responsable par le médecin chargé de l'examen du cadavre, d'effectuer un tri sûr en prévision du recours ou non à l'autorité d'instruction.»

2 Il se peut rarement que le moment du décès soit déterminant soit en matière d'assurance soit en matière de droit successoral. En effet, seul celui ou celle qui survit au défunt a le droit d'hériter. Cela peut paraître banal, mais il existe des exceptions où ça ne l'est pas, notamment si en cas d'héritage, plusieurs personnes décèdent dans un même laps de temps, par exemple lors d'un accident de la route; le moment du décès de chacun peut alors être déterminant pour fixer l'ordre successoral (puisque le concept de mort cérébrale doit être le critère déterminant pour chaque décès, le médecin doit se demander: «Chez quelle personne impliquée dans l'accident la mort cérébrale est-elle survenue en premier?»).

3 En application de l'article 9 de la loi sur la transplantation: «Il s'agira de normes identiques à celles que contiennent les directives de l'ASSM sur la définition et le diagnostic de la mort en vue d'une transplantation d'organes.» (Message concernant la loi sur la transplantation, p. 137). n'existe qu'une seule mort et, juridiquement, elle doit être la même pour tous;

- En revanche, les procédures de constatation du décès peuvent différer selon le contexte; [...]

\subsubsection{Article 9 Critère du décès et constatation du décès \\ [...] \\ L'al. 2 confère au Conseil fédéral la compétence d'édicter des dispositions détaillées sur la constata- tion du décès. Il s'agira de normes identiques à cel- les que contiennent les directives de l'ASSM sur la définition et le diagnostic de la mort en vue d'une transplantation d'organes.» (Message p. 137)}

\section{Le médecin de famille reste compétent pour constater le décès dans un contexte «habituel»}

Marcel Monnier, juriste chargé de la loi sur la transplantation auprès de l'OFSP, nous a confirmé que dans l'ordonnance relative à la loi sur la transplantation, le Conseil fédéral règlera uniquement la question de la constatation du décès en rapport avec la transplantation d'organes. «Quand il ne s'agit pas de transplantations, le décès peut/doit aussi être constaté selon la procédure habituelle par le médecin de famille (et donc bien évidemment pas uniquement par des médecins spécialistes).» [trad. FMH]

Et Monnier de poursuivre: «La mort est la même pour tous les êtres humains, c'est pourquoi cette question doit être réglée de la même manière pour tous sur le plan juridique. Si tel n'était pas le cas, il serait possible qu'une personne entrant en ligne de compte pour un don d'organe soit déclarée morte à un autre moment que si elle était décédée dans un autre contexte, ce qui est absolument inimaginable. Tout médecin, qu'il soit médecin de premier recours ou médecin hospitalier, se base sur les mêmes critères pour constater le décès, mais suivant les circonstances il peut avoir recours à un autre procédé (pour le médecin de famille qui est appelé le lendemain matin pour constater un décès survenu durant la nuit, le constat de la rigidité cadavérique suffit).»

\section{Commentaire}

Le message et la loi seraient plus clairs si la réalité des faits était nommée. En effet, même si l'on arrive à s'entendre sur le plan juridique pour dire qu'il n'existe qu'un seul concept de la mort pour l'ensemble de la Suisse, il est clair qu'aujourd'hui et à l'avenir également, les décès dans le cadre de la transplantation d'organes sont et seront constatés selon d'autres règles que les décès dits «habituels»:

Dans le domaine de la transplantation, les abus doivent être exclus et la confiance de la population assurée. Pour ce faire, la séparation des pouvoirs au sein de l'équipe médicale est nécessaire, de même qu'un examen technique détaillé de la mort cérébrale.

En dehors du contexte de transplantation, c'est-à-dire en cas de décès habituel, le médecin traitant peut et doit constater le décès. Une séparation des pouvoirs dans cette situation n'est donc ni judicieuse ni nécessaire. Il s'agit ici en fin de compte des deux aspects importants suivants: les médecins doivent pouvoir exclure une cause de décès non naturelle [1] de même qu'ils doivent pouvoir déterminer le moment du décès de manière réaliste [2].

\section{Conclusion:}

- Dans l'ordonnance à la loi sur la transplantation, le Conseil fédéral réglera la question de la constatation du décès dans le cadre de la transplantation d'organes.[3]

- En revanche, la réglementation de la constatation du décès «habituel» dans le reste du domaine de la santé incombe encore et toujours entièrement aux cantons. (Cela revient à dire que si, pour la constatation d'un décès en dehors de la médecine de transplantation, un canton faisait référence à la législation fédérale, il s'agirait incontestablement d'une erreur, car celle-ci ne règle justement pas cette question.) 